\title{
Ultra-orphan diseases: a quantitative analysis of the natural history of molybdenum cofactor deficiency
}

\author{
Konstantin Mechler, MD¹, William K. Mountford, PhD², Georg F. Hoffmann, MD³ and \\ Markus Ries, MD, $\mathrm{PhD}^{3}$
}

Purpose: Experimental treatment with substrate replacement was successfully performed in single cases with molybdenum cofactor deficiency type A. The objective of this study was to quantitate the yet undefined natural history in untreated patients to ultimately benefit knowledge in experimental treatments in the future.

Methods: Systematic analysis of published cases with molybdenum cofactor deficiency. The main outcome measures were survival, initial cardinal disease features at onset, and diagnostic delay.

Results: The median survival for the overall population was 36 months. Initial cardinal disease features at onset were seizures (72\%) as well as feeding difficulties (26\%) and hypotonia (11\%). In addition, developmental delay (9\%), hemiplegia (2\%), lens dislocation ( $2 \%$ ), and hyperreflexia (1\%) were reported. The median age at onset of the disease was the first day of life; the median age at diagnosis was 4.5 months. The median time to diagnosis (diagnostic delay) was 89 days.

Conclusion: Molybdenum cofactor deficiency has its onset during the neonatal period and infancy. There is considerable diagnostic delay. Although seizures were the most frequent initial cardinal sign, molybdenum cofactor deficiency should be considered as a differential diagnosis in patients presenting with hypotonia, developmental delay, or feeding difficulties. The survival data will inform further natural-history and therapeutic studies.

Genet Med advance online publication 12 March 2015

Key Words: drug development; molybdenum cofactor deficiency; natural history; orphan disease; survival

\section{INTRODUCTION}

Molybdenum cofactor deficiency (MoCD) is a life-threatening, pan-ethnic, rare autosomal-recessive disorder characterized by the deficiency of three molybdenum-dependent enzymes: sulfite oxidase (SOX), xanthine dehydrogenase, and aldehyde oxidase. While all three enzymes are dependent on molybdenum cofactor, the loss of SOX activity is thought to be responsible for the severe and rapidly progressive neurological damage seen in MoCD. There is currently no evidence that the other oxidases play no role in pathogenesis or disease severity. As SOX degrades sulfur-containing acids and converts sulfite to sulfate, sulfite accumulates in patients with MoCD, which results in an intoxication-type disorder. ${ }^{1,2}$ The molybdenum cofactor necessary for the enzymatic activity of the three detoxifying proteins is synthesized in a four-step biochemical pathway from guanosine triphosphate. ${ }^{3}$ There are three types $(\mathrm{A}, \mathrm{B}$, and $\mathrm{C})$ of MoCD. MoCD type A is caused by a mutation in the molybdenum cofactor synthesis (MOCS) 1 gene localized on 6p21.3, and 32 mutations of the MOCS1 gene have been described. ${ }^{4}$ In MoCD type A, the first of the four synthetic steps in the formation of molybdenum cofactor is interrupted, and guanosine triphosphate cannot be converted into cyclic pyranopterin monophosphate (cPMP). The MOCS2 and gephyrin (GPHN) genes are defective in MoCD type $\mathrm{B}$ and type $\mathrm{C}$, respectively. ${ }^{5,6}$
Of note, MoCD is different from isolated SOX deficiency. Both conditions are biochemically and molecularly distinct entities. This article focuses exclusively on patients with MoCD.

Systematic quantitative natural-history data are not available. Shortly after birth, patients with MoCD usually present signs and symptoms such as intractable tonic-clonic seizures, metabolic acidosis, intracranial hemorrhage, exaggerated startle reactions, axial hypotonia, limb hypertonia, and feeding difficulties. ${ }^{7}$ Neuronal damage is severe and rapidly progressive as a result of the accumulation of toxic concentrations of sulfite in the brain. ${ }^{8}$ Brain imaging studies reveal a diffuse pattern of brain atrophy with arrested development of myelination, evidence of gliosis, and cystic necrosis of cerebral white matter. ${ }^{9}$ Microcephaly is common. Dysmorphic facial stigma can be similar to that in patients with perinatal asphyxia. ${ }^{2}$ Other observed features of MoCD include dilated ventricles, hydrocephalus, brain hypodensity, brain atrophy, spastic paraplegia, myoclonus, and opisthotonus. ${ }^{2}$ Ocular abnormalities include lens dislocation, spherophakia, iris coloboma, nystagmus, and enophthalmos. Cerebral blindness has occurred.

The missing cPMP can be produced in Escherichia coli to replace the missing substrate in individuals with MoCD type A. ${ }^{10}$ cPMP was experimentally substituted in a few patients, and orphan drug designation was granted to this replacement

${ }^{1}$ Pediatric Psychopharmacology, Department of Child and Adolescent Psychiatry and Psychotherapy, Central Institute of Mental Health, Medical Faculty Mannheim, University of Heidelberg, Mannheim, Germany; ${ }^{2}$ Department of Clinical Research, School of Nursing, College of Health and Human Services, University of North Carolina at Wilmington, Wilmington, North Carolina, USA; ${ }^{3}$ Pediatric Neurology and Center for Rare Disorders, Center for Pediatric and Adolescent Medicine, Heidelberg University Hospital,

Heidelberg, Germany. Correspondence: Markus Ries (markus.ries@uni-heidelberg.de) 
therapy by the US Food and Drug Administration in 2009. As single cases have been treated with cPMP and clinical trials may follow, a thorough understanding of the natural history is indispensable to distinguish short-term and long-term treatment effects from the natural course of the condition. However, the natural history has not been studied quantitatively, and systematic natural-history studies have not been conducted. Ideally, specific therapies for MoCoA would prolong life and maintain neurological function. Death commonly occurs in the neonatal period, and patients who survive that period usually develop encephalopathy and developmental delay, but survival with this condition has never been quantified. ${ }^{7,8}$ Because survival is considered a potential hard end point for clinical trials, we directed our efforts toward the following primary research question: What is the median survival for untreated patients with MoCD reported in the medical literature? In addition, because early diagnosis may be a key factor for a better outcome in the future, understanding current diagnostic gaps and barriers is important. We therefore quantitated diagnostic delay as well as the distribution of leading signs and symptoms of the condition.

\section{Literature review and definitions}

\section{MATERIALS AND METHODS}

We conducted a thorough literature search for "molybdenum cofactor deficiency" in Medline. Initially, 113 publications were identified, all but one of which were in English (the exception was in Spanish). Publication dates ranged from June 1980 to January 2013. Only data from patients with MoCD were included. Patients with isolated SOX deficiency were not included. Publications were analyzed for suitable clinical information by collecting the following variables: age at onset, leading signs or symptoms at onset, age at diagnosis, sex, siblings diagnosed with MOCD, last reported age, whether the patient was dead or alive at that point, genotype, biochemical diagnosis, and neuroradiological findings. If time of onset or time of diagnosis was stated in vague terms (such as during the first week after birth), a conservative interpretation was made (e.g., "during the first week" was considered to be at day 7). Missing data were not imputed. Duplicate reports of the same patients or patients who received treatment with cPMP were excluded. The remaining 82 patients, described in 49 publications, were included in our study.

\section{Statistical analysis}

Baseline patient demographics and neuroradiological findings were summarized descriptively using patient counts and percentages of the total population. Furthermore, demographic variables, such as gender and genotype, were compared using $\chi^{2}$ tests for equality of proportions to better describe the overall population. Survival was assessed and defined as the time elapsed between patient birth and time of death. Patient data were censored at the time of last follow-up if the patient was still alive at last contact based on data gathered from each publication. The Kaplan-Meier method was used to describe survival for the entire study population, and the log-rank test was used to evaluate potential differences in survival for patients identified as having MoCD compared with other genotypes. Time to diagnosis was calculated by subtracting the age at diagnosis by the age at onset in a given patient $(n=49)$. Neuroradiological data were available for 52 of 82 patients. Findings as reported in the respective publications were quantitatively analyzed, whereas findings describing the same pathological entity with different wordings were grouped together.

All analyses were performed using SAS (SAS Institute, Cary, NC). $P$ values reported were two-sided, and those $\leq 0.05$ were deemed statistically significant.

\section{Demographics}

\section{RESULTS}

We identified 82 children in this study. Of these patients, 70\% were classified as MoCD not otherwise specified based on clini$\mathrm{cal}$ and biochemical diagnosis in the respective reports. The majority of these cases predated the availability of molecular genetic diagnostics. Information on genotype was available for the remaining $30 \%$ of the sample. About half of those (i.e., $\sim 15 \%$ ) were diagnosed with a MOCS1 mutation and $10 \%$ and $6 \%$ with MOCS2 and GPHN mutations, respectively (Table 1). Both sexes were equally represented.

\section{Survival analysis}

The median survival for the population was 36 months (Figure 1). At the end of follow-up in each of the case reports, 42 of the patients were alive (51\%) and 40 had died (49\%). Figure 2 displays the survival distribution of all patients. A subgroup analysis of genotype was performed. The resulting

Table 1 Demography of the study population with molybdenum cofactor deficiency $(n=82)$

\begin{tabular}{lccc} 
Population characteristics & N & Percentage & $\boldsymbol{P}$ value $^{\mathbf{a}}$ \\
\hline Sex & 34 & 42 & 0.72 \\
Female & 37 & 45 & \\
Male & 11 & 13 & \\
Unknown & & & $<0.001$ \\
Diagnosis & 57 & 70 & \\
MoCD (not otherwise specified) & 12 & 15 & \\
MOCS1 mutation & 8 & 10 & \\
MOCS2 mutation & 5 & 6 & \\
GPHN mutation & & & \\
Affected siblings & 31 & 38 & \\
Yes & 49 & 60 & \\
No & 2 & 2 & \\
Unknown & & & \\
Alive at last follow-up & 42 & 51 & \\
Yes & 40 & 49 & \\
No & &
\end{tabular}

GPHN, gephyrin; MoCD, molybdenum cofactor deficiency.

a $P$ value from $\chi^{2}$ test for equal proportions. 


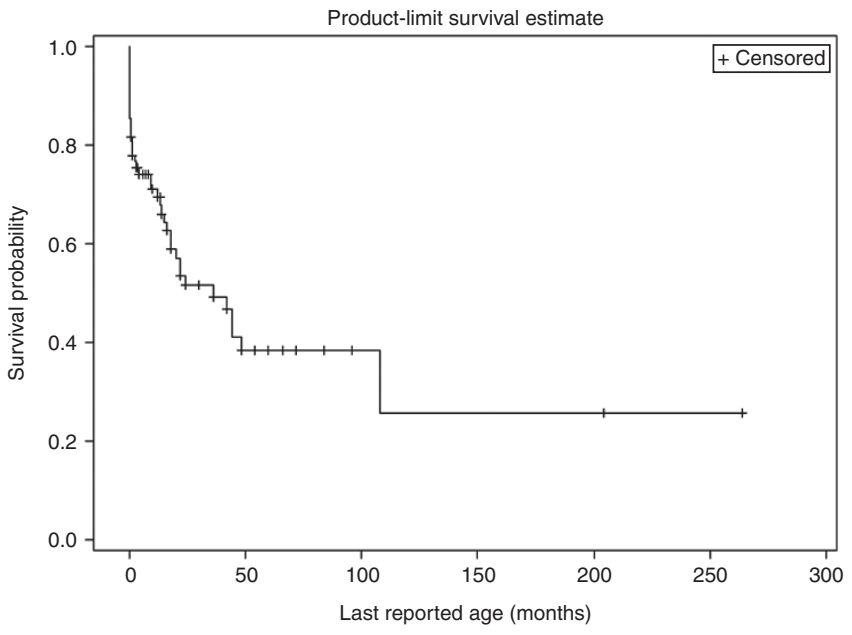

Figure 1 Estimated survival distribution for patients with molybdenum cofactor deficiency among the overall population $(\boldsymbol{N}=\mathbf{8 2})$. Censored individuals are marked with a "+".

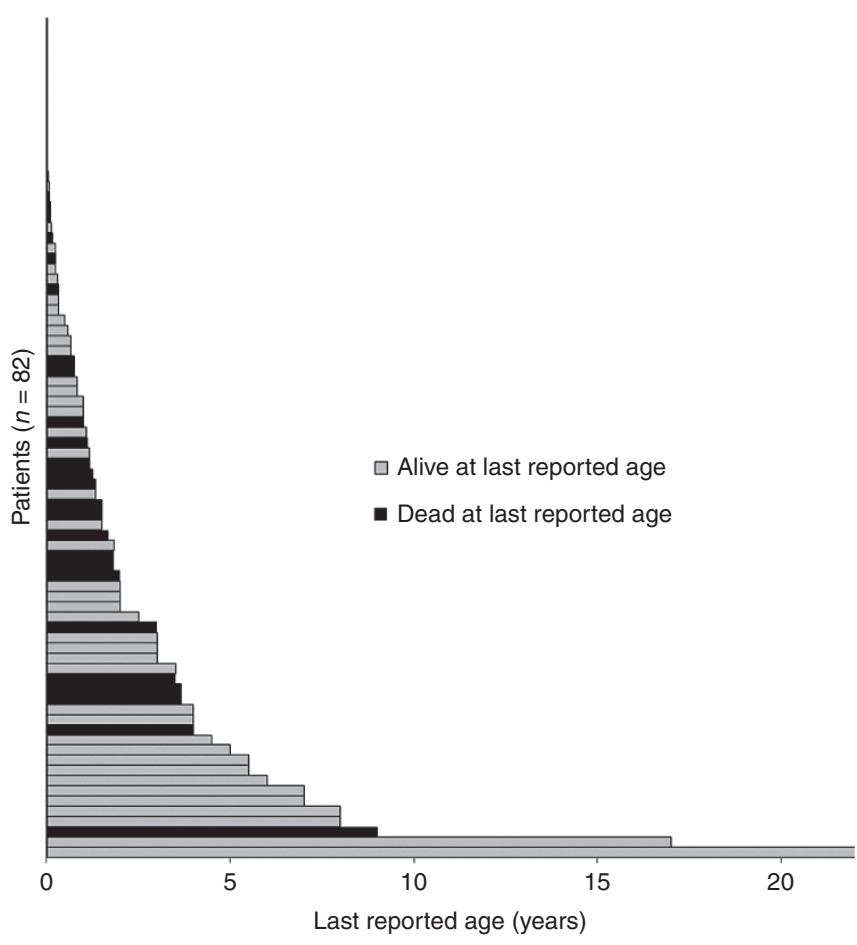

Figure 2 Lifespan of patients with molybdenum cofactor deficiency (black bars indicate deceased patients, gray bars show patients alive at the last follow-up; $n=82$ ).

survival distributions are shown in Supplementary Figure S1 online. The survival curves depicted need to be interpreted cautiously because sample sizes of the subgroups were small, and for many patients included in this study no information on genotype was available at all.

\section{Age at onset and time to diagnosis}

In the overall group $(n=74)$, the median age at onset of the disease was 0.03 months, that is, 1 day (mean 2.3 months; range

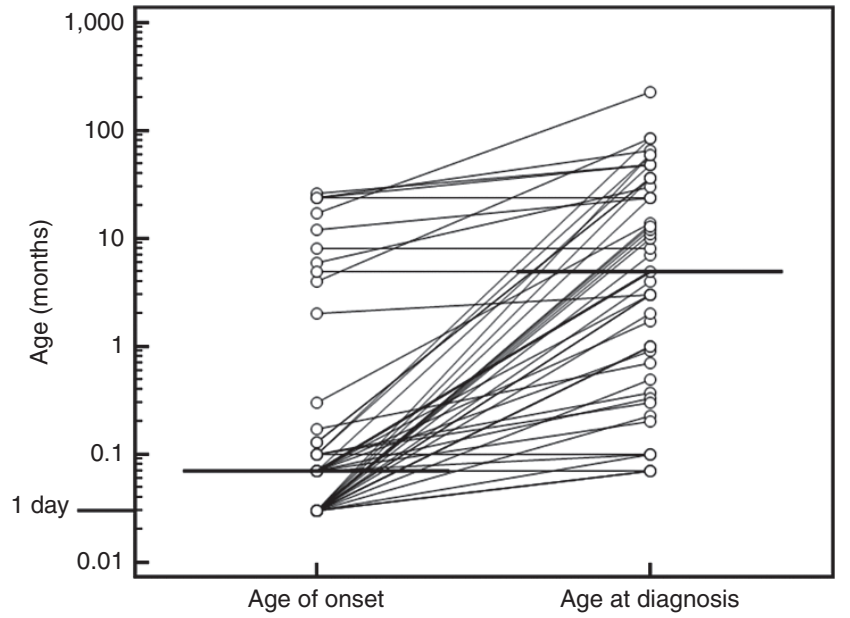

Figure 3 Age at onset (in months) of molybdenum cofactor deficiency and age at diagnosis. Data were available for $n=49$ patients. Horizontal lines indicate the median. The slopes of the connecting lines represent the diagnostic delay between onset of the disease and making the diagnosis.

0.03-26 months). Thirty-eight patients (46\%) showed symptoms on their first day of life, eventually leading to diagnosis of MoCD (Figure 3). Onset of disease within the neonatal period, defined as the 28 days after birth, occurred in 61 patients (73\%). The latest reported onset was at age 26 months ( 2 years 2 months).

We calculated the time to diagnosis for each patient (interval between age at onset of first signs or symptoms and the age at diagnosis; Figure 3). The median time to diagnosis was 89 days (mean, 18.4 months; interquartile range, 23.7 months; range, 0 days to 211 months; $n=49$ ). The longest reported time to diagnosis was 211 months. The median age at diagnosis was 4.5 months (mean, 21.8 months; interquartile range, 32.4 month; range, 2 days to 228 months; $n=52$ ).

\section{Signs and symptoms}

We analyzed the distribution of presenting or leading signs or symptoms in the study population (Table 2). The most common symptoms were seizures, as well as feeding difficulties and hypotonia. In addition, developmental delay, hemiplegia, lens issues, and hyperreflexia were reported. Motor development delay could by nature be found only in patients with a disease manifestation later than within the first weeks after birth.

\section{Neuroradiological findings}

We analyzed the reported neuroradiological findings in the study population ( $n=52 / 82$; Supplementary Table $S 1$ online). More than $40 \%$ of patients had signs of reduced myelination, enlarged ventricles, cerebral atrophy, and cystic lesions. More than $20 \%$ had an atrophy of the corpus callosum, enlarged extra-axial spaces, lesions in the basal ganglia, and cerebellar atrophy.

\section{DISCUSSION}

$\mathrm{MoCD}$ is an orphan neurodegenerative disorder manifesting at birth or during infancy. The condition is of particular interest 
because a specific therapy, that is, substrate replacement therapy with cPMP, is being developed for the most common subtype: MoCD type A.

The median survival in this cohort was 36 months. The majority of patients presented with a neonatal onset at birth. Some patients had a gradually delayed onset of disease, as one would expect for a neurogenetic condition. Not surprising for a rare metabolic condition, there was a diagnostic gap with considerably delayed diagnosis in some individuals. This may be due to the fact that awareness of this orphan disease is low and that biochemical and molecular testing is not always locally available, which creates diagnostic barriers. In some cases siblings were diagnosed within the first days of life, which emphasizes the importance of the family history. There was no evidence of a separate neonatal or late infantile onset because age at onset showed a gradually increasing distribution pattern. Seizures were the most prominent initial sign in the majority of patients and usually led to a metabolic workup. Some presented with other neurological features, however, such as feeding difficulties, hypotonia, and developmental delay. The nature of signs and symptoms at onset were similar in younger and older patients. Developmental delay was not an isolated symptom in 6 of the 7 cases reported. One patient, reported by Arenas et al., ${ }^{11}$ initially presented with developmental delay at the age of 2 years, and lens dislocation was diagnosed at 4 years, while the patient was still seizure-free at the age of 7 years. Therefore, considering MoCD as a differential diagnosis for developmental delay is important; at the same time, we emphasize the importance of an eye examination in the workup. MoCD is a rare disorder, and the condition is probably underdiagnosed because of the relatively difficult clinical suspicion.

The clinical diagnosis of MoCD, often supported by magnetic resonance imaging findings, as shown in Supplementary Table S1 online, has to be confirmed by biochemical and molecular genetic studies. Metabolic findings in MoCD reflect the pattern of enzyme deficiencies of SOX, xanthine dehydrogenase, and aldehyde oxidase. This includes elevated urinary excretion of sulfate, thiosulfate, S-sulfocysteine, taurine, xanthine, and hypoxanthine. Uric acid is low in plasma and urine., ${ }^{2,6,12}$ Elevated xanthine and hypoxanthine concentrations provide a better biochemical signal then uric acid concentrations, which

Table 2 Signs or symptoms initially presenting in patients with molybdenum cofactor deficiency $(n=82)$

\begin{tabular}{lcc} 
Symptom $^{\text {a }}$ & N & Percentage \\
\hline Seizures & 59 & 72 \\
\hline Feeding difficulties & 21 & 26 \\
Hypotonia & 9 & 11 \\
\hline Motor development delay & 7 & 9 \\
Lens dislocation & 2 & 2 \\
\hline Hemiplegia & 2 & 2 \\
Hyperreflexia & 1 & 1 \\
\hline
\end{tabular}

In individual patients, two or more signs or symptoms may have been reported. are not always severely depressed in MoCD. Of note, xanthine, hypoxanthine, and uric acid concentrations are normal in the phenotypically similar but biochemically and molecularly different disorder of isolated SOX deficiency, which is helpful in the differential diagnostic workup. Elevated concentrations of sulfite in the urine can be identified with a dipstick test. However, this test is a bedside test and is often false negative because sulfite is unstable. A better marker for sulfite metabolism is S-sulfocysteine, which is a stable metabolite. ${ }^{2}$ In the present population with available, quantitatively reported urinary S-sulfocysteine data, the concentrations in urine had a median elevation of 30-fold (interquartile range, 10- to 48-fold; range, 2- to 290-fold) (Supplementary Figure S2 online).

The diagnosis of the precise subtype of MoCD is established through molecular genetic analysis of the MOCS or GPHN genes. ${ }^{5}$

Current treatment for individuals with this disorder aims to provide relief of symptoms (e.g., treatment with anticonvulsants for seizures) and support in the care of the patient, such as placement of a feeding tube. Dietary restriction of sulfur-containing amino acids may decrease sulfite excretion but was not able to stop the neurological progression of the disease. ${ }^{13}$

With the potential availability of a specific therapy for $\mathrm{MoCD}$ type A, early diagnosis becomes important because an early intervention has the potential to deliver a potentially better outcome. ${ }^{14}$ In a clinical proof-of-concept experiment using one patient, the missing metabolite cPMP was replaced with a purified compound synthesized in E. coli. In an infant diagnosed at 6 days of life, the daily i.v. substitution with purified cPMP, started on day 36 , led to a decrease of urinary disease markers (i.e., sulfite, S-sulfosysteine, thiosulfate), and xanthine as well as uric acid returned to almost normal concentrations, while the child showed clinical improvements in epileptic seizures. ${ }^{6}$ A second case of successful E. coli-derived cPMP substitution therapy was recently described by Hitzert et al. ${ }^{15}$ This infant was prenatally diagnosed with MoCD type A, birth was induced at $36+3$ weeks of gestation, and treatment with E. coli-derived cPMP commenced $4 \mathrm{~h}$ after birth. Biomarkers normalized within days 2-16. At 21 months, behavioral problems could not be detected. Fine motor, gross motor, and total motor development were normal. Cognitive development was only mildly delayed. Except for three central line infections, the authors reported that no serious drug-related adverse effects occurred. ${ }^{15}$ The efficient uptake of cPMP and restoration of molybdenum cofactor-dependent enzyme activities were reported in six other patients by Veldman et al. ${ }^{16}$ These newborns had been diagnosed with MoCo type A in utero or at the age of 2-20 days. E. coli-derived cPMP substitution treatment was initiated on days $0-36$. Biomarkers of SOX (sulfite, S-sulfocysteine, thiosulfate) and xanthine oxidase deficiency (xanthine, uric acid) returned to almost normal within days. All infants became more alert, convulsions and twitching disappeared within the first 2 weeks, and the electroencephalogram showed the return of rhythmic elements and markedly reduced epileptiform discharges. ${ }^{16}$ Schwahn et al. ${ }^{17}$ reported the efficacy of long-term E. coli-derived cPMP substitution in two 
unrelated children with MoCD type A. Diagnoses were made on day 1 of life in baby 1 because of a previously affected sibling and on day 4 in baby 2 , who started having intractable seizures from day 1 of life. Daily i.v. cPMP infusions were started on day 7 and 5 of life, respectively. Both infants tolerated cPMP without adverse effects, apart from intercurrent central venous line infections. The authors observed a rapid and sustained good clinical and biochemical response. Baby 1 showed satisfying developmental progress, whereas baby 2 , despite earlier treatment, suffered from static, dystonic cerebral palsy, and cystic encephalopathy caused by early, nonprogressive necrotic changes, probably predating cPMP substitution. ${ }^{17}$ Earlier intervention might deliver better outcomes, but this requires further study. The potential necessity of early treatment would render timely diagnosis crucial.

This study has some limitations. Some cases are not genetically classified because molecular analysis was not available at the time of publication. The analysis is therefore pooled for all types of MoCD. Because all types of MoCD result in the dysfunction of the same three enzymes, there might not be a substantial clinical difference between the different subtypes of MoCD from a pathophysiological point of view, but this statement contains some uncertainty in the absence of a definite proof. Therefore, we provide survival date by (i) MoCD subtypes and (ii) for the pooled group. The survival of more recent cases may have improved by now because the standard of care has changed. Because there are reports from many regions all over the world, the comparison of survival between two different regions may be limited because of different standard of care. However, the presence of global patients enriches the diversity of the database. With the present methodology the analysis of soft end points, such as development, neurocognitive outcome, or quality of life, is very difficult because of the lack of standardized ascertainment and the plethora of available measurement instruments. The analysis of the neuroradiological findings is limited because of the pooling of data that were ascertained at various ages, different methods of imaging and machines, the absence of a centralized blinded reading, and the lack of a standardized protocol. As such, some publications mention only the key findings, whereas others include the complete results. The S-sulfocysteine values depicted in Supplementary Figure S2 online. were assessed in different laboratories. This study is useful, however, in the quantitation of hard end points such as survival time, and therefore these data will be instrumental in planning further clinical research. Other methods for natural-history studies such as questionnaire-based study would entail recall bias..$^{18} \mathrm{~A}$ retrospective chart review may be subject to ascertainment bias, and the evolving standard of care over time is difficult to account for in such a setting. Ideally, the natural history is studied in a prospective way, which implies a long duration. Imaging data should be assessed by standardized protocol and blinded central readers. Whereas biomarkers are useful in the assessment of specific therapies, the availability of laboratory samples renders this feasible in a prospective natural-history setting only. Natural-history data are obtained only once therapy is available, such as in Fabry disease, and this is a common phenomenon. The issues of selection bias, ascertainment bias, and missing data can render the comparison of treatment effects versus natural history difficult. ${ }^{19}$ Another approach is the comparison of natural-history patients from a database with treated patients in a matched pair design. ${ }^{20}$

In a rare condition such as $\mathrm{MoCD}$, close collaboration among all stakeholders is important because collaborative studies and systematic global data collection become a major effort. As a next step, a global retrospective natural-history study based on chart review will further enhance the understanding of longitudinal hard end points over a long period, whereas a prospective natural-history study can help us to better understand biomarkers and test the feasibility of instruments measuring development, cognition, and quality of life relevant for future therapeutic clinical trials.

\section{SUPPLEMENTARY MATERIAL}

Supplementary material is linked to the online version of the paper at http://www.nature.com/gim

\section{ACKNOWLEDGMENTS}

Part of this research was previously published in abstract form as a contribution to the "Neurowoche" conference (Munich, Germany, 2014), in Neuropediatrics 2014;45(suppl 1):S12.

\section{DISCLOSURE}

M.R. received consultancy fees from Alexion Pharmaceuticals. The other authors declare no conflict of interest.

The authors have no financial relationships relevant to this article to disclose, and no funding was secured for this study.

\section{REFERENCES}

1. Reiss J, Bonin M, Schwegler H, et al. The pathogenesis of molybdenum cofactor deficiency, its delay by maternal clearance, and its expression pattern in microarray analysis. Mol Genet Metab 2005;85:12-20.

2. Johnson JL, Duran M. Molybdenum cofactor deficiency and isolated sulfite oxidase deficiency. In: Scriver CR, Beaudet AL, Sly WS, Valle D (eds). The Metabolic and Molecular Bases of Inherited Disease, 8th edn. McGraw-Hill: New York, 2001:3163-3177.

3. Schwarz G. Molybdenum cofactor biosynthesis and deficiency. Cell Mol Life Sci 2005;62:2792-2810.

4. The Human Gene Mutation Database at the University of Cardiff. Molybdenum Cofactor Synthesis 1. http://www.hgmd.cf.ac.uk/ac/gene.php?gene=MOCS1. Accessed 1 October 2014

5. Reiss J, Hahnewald R. Molybdenum cofactor deficiency: mutations in GPHN MOCS1, and MOCS2. Hum Mutat 2011;32:10-18.

6. Veldman A, Santamaria-Araujo JA, Sollazzo S, et al. Successful treatment of molybdenum cofactor deficiency type A with CPMP. Pediatrics 2010;125: e1249-e1254.

7. van der Knaap M, Valk J. Molybdenum cofactor deficiency and isolated sulfate oxidase deficiency. Magnetic resonance of myelination and myelin disorders, 3rd edn. Springer: New York, 2005:372-376.

8. Per H, Gümüş H, Ichida K, Cağlayan $\mathrm{O}$, Kumandaş S. Molybdenum cofactor deficiency: clinical features in a Turkish patient. Brain Dev 2007;29: 365-368.

9. Appignani BA, Kaye EM, Wolpert SM. CT and MR appearance of the brain in two children with molybdenum cofactor deficiency. AJNR Am J Neuroradiol 1996;17:317-320.

10. Schwarz G, Santamaria-Araujo JA, Wolf S, et al. Rescue of lethal molybdenum cofactor deficiency by a biosynthetic precursor from Escherichia coli. Hum Mol Genet 2004;13:1249-1255. 
11. Arenas M, Fairbanks LD, Vijayakumar K, Carr L, Escuredo E, Marinaki AM. An unusual genetic variant in the MOCS1 gene leads to complete missplicing of an alternatively spliced exon in a patient with molybdenum cofactor deficiency. J Inherit Metab Dis 2009;32:560-569.

12. Belaidi AA, Schwarz G. Molybdenum cofactor deficiency: metabolic link between taurine and S-sulfocysteine. Adv Exp Med Biol 2013;776: 13-19.

13. Boles RG, Ment LR, Meyn MS, Horwich AL, Kratz LE, Rinaldo P. Short-term response to dietary therapy in molybdenum cofactor deficiency. Ann Neurol 1993;34:742-744.

14. Veldman A, Hennermann JB, Schwarz G, et al. Timing of cerebral developmental disruption in molybdenum cofactor deficiency. J Child Neurol 2011;26: 1059-1060; author reply 1061.

15. Hitzert MM, Bos AF, Bergman KA, et al. Favorable outcome in a newborn with molybdenum cofactor type A deficiency treated with CPMP. Pediatrics 2012;130:e1005-e1010.
16. Veldman A, Schwahn BC, Galloway J, et al. Efficacy and safety of cyclic pyranopterin monophosphate in the treatment of six newborn babies with molybdenum cofactor deficiency type A. J Inherit Metab Dis 2011;34(suppl):84. Abstract number P-023.

17. Schwahn BC, Galloway J, Bowhay S, et al. Follow-up of two infants with molybdenum cofactor deficiency (MOCD) group A, on long-term treatment with cyclic pyranopterin monophosphate (cPMP). J Inherit Metab Dis 2011;34(suppl):84. Abstract number P-024.

18. Bley AE, Giannikopoulos OA, Hayden D, Kubilus K, Tifft CJ, Eichler FS. Natural history of infantile G(M2) gangliosidosis. Pediatrics 2011;128:e1233-e1241.

19. Mehta A, Beck M, Elliott P, et al.; Fabry Outcome Survey investigators. Enzyme replacement therapy with agalsidase alfa in patients with Fabry's disease: an analysis of registry data. Lancet 2009;374:1986-1996.

20. Weidemann F, Niemann M, Störk S, et al. Long-term outcome of enzymereplacement therapy in advanced Fabry disease: evidence for disease progression towards serious complications. J Intern Med 2013;274:331-341. 